\title{
Association between the degree of fibrosis in fibrotic focus and the unfavorable clinicopathological prognostic features of breast cancer
}

\author{
Yongfu Li ${ }^{1,2}$, Yuhan Wei ${ }^{1}$, Wenjun Tang ${ }^{2}$, Jingru Luo ${ }^{2}$, Minghua Wang ${ }^{3}$, Haifeng Lin ${ }^{2}$, Hong Guo ${ }^{4}$, Yuling Ma $^{5}$, \\ Jun Zhang ${ }^{6}$, Qin Li ${ }^{\text {Corresp. } 1}$ \\ ${ }^{1}$ Department of Oncology, Beijing Friendship Hospital, Capital Medical University, Beijing, China \\ 2 Department of Oncology, The Second Affiliated Hospital of Hainan Medical University, Haikou, Hainan Province, China \\ 3 Department of Pathology, The Second Affiliated Hospital of Hainan Medical University, Haikou, Hainan Province, China \\ 4 Department of Surgery, Beijing Changping District Hospital of Traditional Medicine, Beijing, China \\ 5 Department of Gynecology, Beijing Changping District Hospital of Traditional Medicine, Beijing, China \\ 6 Department of Hematopathology, University of Texas MD Anderson Cancer Center, Houston, TX, US \\ Corresponding Author: Qin Li \\ Email address: oncologistinbj@163.com
}

Objective. To explore the association between the degree of fibrosis in fibrotic focus (FF) and the unfavorable clinicopathological prognostic features of breast cancer. Methods. 169 cases of breast invasive ductal carcinoma (IDC) were included in the study. Hematoxylin and eosin (H\&E) staining was performed in the primary lesion of breast IDC and the degree of fibrosis in tumor-stromal FF was assessed. The association between the degree of fibrosis in FF and the well-known clinicopathologicfeatures of breast cancer was investigated and the influence of the degree of fibrosis in FF on the survival was analyzed. Results. Tumor size $>2 \mathrm{~cm}(P=0.023)$, vascular invasion $(P=0.011)$, lymphatic vessel invasion $(P<0.001)$ and HER-2+ $(P=0.032)$ were positively correlated with the degree of fibrosis in FF in breast IDC. The result of multivariate analysis showed that lymphatic vessel invasion was the only independent correlation factor of high fibrosis in FF in breast IDC (OR $=3.82,95 \% \mathrm{Cl} 1.13 \sim 12.82, P=0.031)$. The Nottingham prognostic index (NPI) of high fibrosis in FF was significantly higher than that of mild and moderate fibrosis in FF in the no vascular infiltration subgroup, the no nerve infiltration subgroup, and the Luminal $A$ subgroup ( $P=0.014,0.039$, and 0.018 ; respectively). Conclusions. The high fibrosis in FF is closely associated with the strong invasiveness and the high malignancy of breast IDC. The degree of fibrosis in FF might be considered as a very practical and meaningful pathological feature of breast cancer. 
1 Association between the degree of fibrosis in fibrotic

2 focus and the unfavorable clinicopathological

3 prognostic features of breast cancer

4 Yongfu Li ${ }^{1,2}$, Yuhan Wei ${ }^{1}$, Wenjun Tang ${ }^{2}$, Jingru $\mathrm{Luo}^{2}$, Minghua Wang ${ }^{3}$, Haifeng Lin ${ }^{2}$, Hong

$5 \mathrm{Guo}^{4}$, Yuling $\mathrm{Ma}^{5}$, Jun Zhang ${ }^{6}$, Qin $\mathrm{Li}^{1}$

$6{ }^{1}$ Department of Oncology, Beijing Friendship Hospital, Capital Medical University, Beijing,

7 China.

8 2Department of Oncology, The Second Affiliated Hospital of Hainan Medical University,

9 Haikou, Hainan Province, China.

${ }^{3}$ Department of Pathology, The Second Affiliated Hospital of Hainan Medical University, Haikou, Hainan Province, China.

${ }^{4}$ Department of Surgery, Beijing Changping District Hospital of Traditional Medicine, Beijing, China.

${ }^{5}$ Department of Gynecology, Beijing Changping District Hospital of Traditional Medicine, Beijing, China.

${ }^{6}$ Department of Hematopathology, University of Texas MD Anderson Cancer Center, Houston, TX, US.

Corresponding Author:

Qin Li

No.95, Yongan Road, Xicheng District, Beijing, 100050, China.

E-mail address: oncologistinbj@163.com

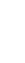

(1)

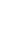

8

29




\section{Abstract}

Objective. To explore the association between the degree of fibrosis in fibrotic focus (FF) and the unfavorable clinicopathological prognostic features of breast cancer.

Methods. 169 cases of breast invasive ductal carcinoma (IDC) were included in the study. Hematoxylin and eosin (H\&E) staining was performed in the primary lesion of breast IDC and the degree of fibrosis in tumor-stromal FF was assessed. The association between the degree of fibrosis in FF and the well-known clinicopathologic features of breast cancer was investigated and the influence of the degree of fibrosis in FF on the survival was analyzed.

Results. Tumor size $>2 \mathrm{~cm}(P=0.023)$, vascular invasion $(P=0.011)$, lymphatic vessel invasion $(P<0.001)$ and HER-2+ $(P=0.032)$ were positively correlated with the degree of fibrosis in FF in breast IDC. The result of multivariate analysis showed that lymphatic vessel invasion was the only independent correlation factor of high fibrosis in FF in breast IDC (OR $=3.82,95 \%$ CI 1.13 12.82, $P=0.031$ ). The Nottingham prognostic index (NPI) of high fibrosis in FF was significantly higher than that of mild and moderate fibrosis in FF in the no vascular infiltration subgroup, the no nerve infiltration subgroup, and the Luminal A subgroup $(\mathrm{P}=0.014,0.039$, and 0.018; respectively).

Conclusions. The high fibrosis in FF is closely associated with the strong invasiveness and the high malignancy of breast IDC. The degree of fibrosis in FF might be considered as a very practical and meaningful pathological feature of breast cancer.

\section{Introduction}

Breast cancer is the most common female malignant tumor across the world (DeSantis et al. 2017). The prognosis of breast cancer is closely associated with various clinicopathological characteristics, such as age, tumor size, pathological type, lymph node metastasis status, histological grade, lymphovascular invasion, Ki-67 index, hormone receptor (HR) status, human epidermal growth factor receptor-2 (HER-2) expression, etc. These well-known pathological features have been widely used to make clinical treatment plans and predict the prognosis of breast cancer. However, the pathophysiology of breast cancer is not only closely related to the tumor cells, but also related to the tumor microenvironment which is composed of sromal cells, infiltrating immune cells, vasculature, extracellular matrix, and various cell signaling factors. Tumor microenvironment plays an important role in the genesis, development, invasion, metastasis, immune escape and chemotherapy resistance of tumor (Reisfeld 2013). FF is a pathological change in the tumor microenvironment of breast cancer, with an incidence of 18.753.0\% (Colpaert et al. 2001; Hasebe et al. 1996; Kornegoor et al. 2012; Mujtaba et al. 2013; Van 
68

den Eynden et al. 2008). Hasebe described the pathologic features of FF in the breast cancer stroma in detail for the first time: a fibrotic lesion, with an appearance of scar or radiating expanding fibrosclerotic core, almost located in the center of carcinoma and consisted of variable amounts of collagen fibers and fibroblasts (Hasebe et al. 1996). At present, it is believed that the formation of FF is driven mostly by intratumoral hypoxia, which reflects the malignancy of carcinoma. Thus, FF is considered as a very practical and easily assessable clinicopathological parameter in breast cancer (Baak et al. 2005; Hasebe et al. 2000; Hasebe et al. 2002; Hasebe et al. 1997; Maiorano et al. 2010; Van den Eynden et al. 2007). According to the variable proportions of collagen fibers and fibroblasts, the degree of fibrosis in FF is classified into three categories: mild, moderate, and high (Hasebe et al. 1996; Van den Eynden et al. 2007). Lots of research fruits have been presented in the correlation between FF and the poor outcome in breast cancer. However, little is known about the association between the degree of fibrosis in FF and the prognosis of breast cancer so far. Whether the degree of fibrosis in FF could better reflect the prognosis of breast cancer than FF has become a research hotspot. Therefore, a retrospective study was conducted to explore the association between the degree of fibrosis in FF and the unfavorable clinicopathological prognostic features of breast cancer.

\section{Materials and methods}

Cases.

The objects of this study were 169 patients with primary breast cancer who underwent surgeries between January 1, 2016 and December 31, 2018 at the Second Affiliated Hospital of Hainan Medical University. Inclusion criteria: (1) female diagnosed with primary breast invasive ductal carcinoma (IDC); (2) IDC must be the principal component ( $>50 \%$ ) in mixed pathological type; (3) no distant metastasis; (4) no neoadjuvant therapy before surgery; (5) FF stained with H\&E was present in the primary lesion of breast cancer. Exclusion criteria: (1) male breast cancer; (2) infiltrative specific breast cancer; (3) secondary carcinoma of mammary gland. All included cases were followed up once every three months by telephone.

\section{Ethical.}

The Second Affiliated Hospital of Hainan Medical University granted Ethical approval to carry out the study within its facilities (Application Ref: LW005). Our Institutional Review board waived the written consent, and the verbal consent was used.

Materials.

Personal information of included cases was collected accurately, including age, family tumor history, smoking history, and drinking history. The clinicopathological features were evaluated based on the pathological reports and the medical records, including tumor size, regional lymph 
103

104

105

106

107

108

109

110

111

112

113

114

115

116

117

118

119

120

121

122

123

124

125

126

127

128

129

130

131

132

133

134

135

136

137

138

node metastasis status, stage, histological grade, vascular invasion, lymphatic vessel invasion, nervous infiltration, estrogen receptor (ER) status, progesterone receptor (PR) status, ki-67 index, HER-2 expression, etc. The measurement of tumor size was based on the largest diameter of invasive component in histological sections. The largest diameter of metastatic lesion > $0.2 \mathrm{~mm}$ in axillary lymph node was defined as regional lymph node metastasis (Plichta et al. 2018). Disease stage was assessed according to the eighth edition of American Joint Committee on Cancer Staging Manual for breast cancer (Plichta et al. 2018). Histological grade was evaluated according to Nottingham modification of the Scarff-Bloom-Richardson histological grading system for invasive breast cancer (Elston \& Ellis 1993). Stained with immunohistochemistry (IHC), the ER expression rate of tumor cells $\geq 1 \%$ was defined as positive ER expression (ER+), otherwise it was defined as negative ER expression (ER-). This criterion also was applied to the evaluation of the PR expression ( $\mathrm{PR}+/-)$. The positive PR expression was classified into two categories: low ( $<20 \%)$ and high $(\geq 20 \%)$ (Prat et al. 2013). Positive hormone receptor expression $(\mathrm{HR}+)$ was defined by ER+ or/and PR + . In the selected area of slides with $>500$ tumor cells, the percentage of tumor cells with positive Ki-67 expression (IHC) was defined as Ki-67 index. Ki-67 index was also classified into two categories: low $(\leq 14 \%)$ and high ( $>14 \%$ ) (Goldhirsch et al. 2011). HER-2 expression status was defined according to the recommendations for HER-2 testing in breast cancer and classified into two categories: positive (HER-2+) and negative (HER-2-) (Wolff et al. 2013). Combining the immunohistochemical findings of ER, PR, HER-2, and Ki-67 index, breast cancer was classified into four molecular subtypes including Luminal A, Luminal B, HER-2 overexpression, and triple negative breast cancer (TNBC). NPI is a widely accepted clinicopathological scoring system for early breast cancer prognostication. NPI $=$ Tumor size $(\mathrm{cm}) \times 0.2+$ Histological grade $(1 \sim 3)+$ Lymph node stage (1 3) (Lee \& Ellis 2008). Histological grade 1 3 was scored as 1 3 points, respectively. Lymph node stage 1 meant no node involved. Lymph node stage 2 meant 1 3 low axillary nodes involved or internal mammary node involved. Lymph node stage 3 meant $\geq 4$ low axillary nodes involved and/or the apical axillary nodes involved or both low axillary nodes and internal mammary nodes involved. Lymph node stage 1 3 was scored as 1 3 points, respectively.

Methods.

All specimens of the primary lesion of breast cancer were fixed in $10 \%$ formalin and cut into $5 \mu \mathrm{m}$-thick sections which were stained with $\mathrm{H} \& \mathrm{E}$ and analyzed by microscopic examination. Firstly, the location, size, appearance and components of FF should be observed under low power microscope. FF was mostly located in the center of the tumor. The size of FF was $\geq 1 \mathrm{~mm}$, otherwise it could not be defined as FF. FF usually appeared as scar-like lesion (Fig. 1A) or irregular moth-eaten radiating fibrosclerotic core (Fig. 1B). Numerous tumor cells could be 
139

140

141

142

143

144

145

146

147

148

149

150

151

152

153

154

155

156

157

158

159

160

161

162

163

164

165

166

167

168

169

170

171

\section{Results.}

173 General characteristics of all cases. agree on the conclusion.

Statistical analysis.

observed frequently around FF. Besides, linear-growing tumor cells and tumor nests could be usually found in FF with diameter $>3 \mathrm{~mm}$. Coagulative necrosis could be present in some FFs. But coagulative necrosis without collagen fiber deposition and fibroblast proliferation was insufficient to be called FF. Sometimes, haemorrhage could be found in FF. Secondly, the components of FF were observed under high power microscope. FF was mainly composed of different proportions of fibroblasts and collagen fibers. The collagen fibers stained with H\&E were thick and arranged closely in bundle. Different amounts of fibroblasts proliferating abnormally could be found among the collagen fibers. In addition, the densities of micro-vessels and micro-lymphaticvessels in FF were significantly higher than those in normal tissue. Evaluating the degree of fibrosis in FF is the last but most important step. According to the proportion of fibroblasts and collagen fibers, FF was classified into three semi-quantitative categories (Van den Eynden et al. 2007): (1) mild fibrosis meant that FF consisted of a large number of fibroblasts and small amount of collagen fibers; (2) moderate fibrosis intermediated mild fibrosis and high fibrosis; (3) high fibrosis meant that FF was mainly composed of collagen fibers. Two experienced pathologists were involved in pathological examination and the other senior pathologist should reassess the degree of fibrosis in $\mathrm{FF}$ when the two pathologists did not

Statistical analyses were performed using IBM SPSS statistics software version 25.0. The mean age and the mean NPI were described by $\overline{\mathrm{X}} \pm \mathrm{SD}$. Qualitative data of general and clinicopathologic features were described by case number, rate, and constituent ratio. Lower quartile $\left(P_{25}\right)$, median $(\mathrm{M})$, and upper quartile $\left(P_{75}\right)$ were used to describe the distribution of NPI variables in different degrees of fibrosis in FF. The associations between the degree of fibrosis in FF and clinicopathologic features were analyzed by Mann-whitney U test and JonckheereTerpstra test. After stratificating the patients by clinicopathologic features, most NPI variables were skewed distribution. So Mann-whitney $U$ test was performed to analyze the difference of NPI variables between mild and moderate fibrosis in FF and high fibrosis in FF. The factors significantly associated with the degree of fibrosis in FF in the univariate analyses $(P<0.05)$ were entered together into ordinal logistic regression analysis. The remaining factors in the multivariate analysis were significant at $P<0.05$. Generalized linear model was used to calculate the odds ratio (OR) and $95 \%$ confidence interval $(95 \% \mathrm{CI})$. All analyses were two-sided and $P<$ 0.05 was considered to indicate a statistically significant difference.

PeerJ reviewing PDF | (2019:08:39938:1:1:NEW 11 Oct 2019) 
174 Of the 169 cases, the mean age was $51.6 \pm 10.0$ (range, 28.0-80.0). 6 cases (3.6\%) had family

175 tumor histories, 21 cases (12.4\%) had drinking histories, and none had smoking history.

176 Clinicopathological characteristics of all cases.

177 The main pathological pattern was IDC. 13 cases (7.7\%) were mixed with one kind of infiltrative 178 specific breast cancers, including mucinous carcinoma (3 cases, 1.8\%), lobular carcinoma (2 179 cases, $0.12 \%$ ), invasive papillary carcinoma ( 2 cases, $0.12 \%$ ), apocrine carcinoma ( 2 cases, $1800.12 \%)$, medullary carcinoma ( 1 case, $0.6 \%$ ), neuroendocrine carcinoma ( 1 case, $0.6 \%)$,

181

182

183

184

185

186

187

188

189

190

191

192

193

194

195

196

197

198

199

200

201

202

203

204

205

206

207

208

209 pleomorphic carcinoma ( 1 case, $0.6 \%$ ), and basal carcinoid carcinoma ( 1 case, $0.6 \%$ ). Other clinicopathologic characteristics are presented in Table 1.

The degree of fibrosis in FF.

FF had been observed in the primary lesion of each case. 11 cases $(6.5 \%), 65$ cases $(38.5 \%)$, and 93 cases $(55.0 \%)$ were evaluated as mild, moderate, and high fibrosis, respectively.

Representative histology of FF in breast IDC is shown in Fig. $1 C \sim E$.

Correlation between the clinicopathological characteristics and the degree of fibrosis in FF.

Tumor size $>2 \mathrm{~cm}$, vascular invasion, and lymphatic vessel invasion were significantly correlated with high fibrosis in FF (Fig. $1 F$ and Fig. $2 A \sim C$ ). Age, lymph node metastasis, stage,

histological grade, and nerve infiltration were not significantly associated with the degree of fibrosis in FF. Correlation between the clinicopathological characteristics and the degree of fibrosis in FF is presented in the Table 2.

Association between molecular subtypes and degree of fibrosis in FF.

Significant association was observed between HER-2+ and high fibrosis in FF $(P=0.032)$ and shown in Fig. 2D and Table 3. The other sub-factors of molecular subtypes (ER, PR, and Ki-67 index) and molecular subtypes were not significantly associated with the degree of fibrosis in FF (Table 3).

Multivariate analysis.

The factors, including tumor size, vascular invasion, lymphatic vessel invasion, and HER-2 expression status, were entered into the ordinal logistical analysis. Due to the missing data of clinicopathological features, 85 cases $(50.3 \%)$ were excluded and 84 cases $(49.7 \%)$ with complete data were finally included in the ordinal logistic regression analysis. The multivariate analysis result indicated that lymphatic vessel invasion was the only independent correlated factor of high fibrosis in FF $(\mathrm{OR}=4.10,95 \%$ CI $1.23 \sim 13.70, P=0.021)$. Comparison of NPI variables between mild and moderate fibrosis in FF and high fibrosis in FF. A total of 161 patients with complete data were eligible for NPI scoring. The mean NPI was $4.60 \pm 1.40$. Due to the small number of patients $(6.5 \%)$ with mild fibrosis in FF, we combined the patients with mild fibrosis and those with moderate fibrosis into one group. Finally, we divided the 161 cases into two groups, mild and moderate fibrosis group and high fibrosis group. No 
210 significant difference was found between the two groups $(Z=-1.862, P=0.063)$ (Fig. 3). The

211 cases were stratificated by the clinicopathological characteristics to further analyze the

212 differences of NPI variables between mild and moderate fibrosis group and high fibrosis group.

213 In the no nerve infiltration subgroup, the no vascular infiltration subgroup, and the Luminal A

214 subgroup, the NPI variables of high fibrosis in FF were significantly higher than those of mild

215 and moderate fibrosis in FF ( $P=0.039,0.014$, and 0.018; respectively) (Table 4 and Fig. 3).

216

\section{Discussion}

218 Our research found that tumor size $>2 \mathrm{~cm}$, vascular invasion, lymphatic vessel invasion, and HER-2+ were positively correlated with the degree of fibrosis within FF in breast IDC by univariate analysis. Moreover, lymphatic vessel invasion was the only independent correlation factor of high fibrosis in FF in breast IDC by multivariate analysis. Although there was no significant difference between the NPI of mild and moderate fibrosis in FF and that of high fibrosis in FF, further analysis showed that the NPI variables of high fibrosis in FF were significantly higher than those of mild and moderate fibrosis in FF in the no vascular infiltration subgroup, the no nerve infiltration subgroup, and the Luminal A subgroup. The above results indicated that the high fibrosis of FF was closely correlated with the aggressive clinicopathological characteristics of breast IDC and reflected the poor outcome of breast IDC with no vascular infiltration, no nerve infiltration or Luminal A subtype.

Hypoxia is the main cause of the fibrosis in tumor stroma (Daniel et al. 2019; Hoffmann et al. 2018). In hypoxia state, tumor cells secrete platelet derived growth factor (PDGF), transforming growth factor- $\beta$ (TGF- $\beta$ ), and fibroblast growth factor-2 (FGF-2). All these cell factors act on the fibroblasts together, resulting in the deposition and remodeling of extracellular matrix and the graduate formation of FF in the tumor stroma. Besides, PDGF, TGF- $\beta$, and FGF-2, together with vascular endothelia growth factor (VEGFR) secreted by fibroblasts and tumor associated macrophages, promote the angiogenesis, lymphangiogenesis, and lymphovascular invasion (Shimada et al. 2017; Van den Eynden et al. 2007). The carcinoma-associated fibroblasts (CAFs), which are the main tumor interstitial cells and transforms from fibroblasts, result in the deposition of collagen and fibronectin in the extracellular stroma. It is considered that CAFs play an important function in the formation of FF and are closely related to the high malignancy of tumor (Balachander et al. 2018; Eiro et al. 2018; Reisfeld 2013; Yang et al. 2016). In addition, the fibrosis can inhibit $T$ lymphocytes infiltration in tumor stroma and resist tumor immunity (Salmon et al. 2012). Therefore, FF forming in tumor stroma demonstrates the high aggressiveness of carcinoma (Van den Eynden et al. 2008). The tumor interstitial fibrosis associated with the adverse prognosis and anti-tumor drug resistance (Grasso et al. 2017) 
245

246

247

248

249

250

251

252

253

254

255

256

257

258

259

260

261

262

263

264

265

266

267

268

269

270

271

272

273

274

275

276

277

278

279

280

has been observed in the carcinoma of breast cancer, pancreatic cancer (Thomas \& Radhakrishnan 2019), colorectal cancer (Ikuta et al. 2018), and gastric cancer.

Hasebe et al (Hasebe et al. 1996) demonstrated that the histological grade and lymph node metastasis rate were higher in breast cancer with FF, especially in that with tumor size $<5 \mathrm{~cm}$. Jeong et al (Jeong et al. 2018) revealed that the FF was significantly associated with tumor size > $2 \mathrm{~cm}$, lymph node metastasis, poor differentiation, and vascular invasion in breast cancer. The studies mentioned above had indicated that FF was closely related with the adverse pathological characteristics of breast cancer. However, previous studies had rarely reported the association between the degree of fibrosis in FF and the pathological features of breast cancer, so we conducted this research. Our study found that the degree of fibrosis within FF in breast IDC with tumor size $>2 \mathrm{~cm}$ was significantly higher than that with tumor size $\leq 2 \mathrm{~cm}$, indicating the more severe hypoxia occurred in larger tumors. Besides, our study also revealed that the degree of fibrosis within FF was significantly higher in breast cancer with vascular infiltration and lymphatic infiltration. It might be that the densities of blood vessels and lymphatics were higher in the tumor with high degree fibrosis of FF, increasing the probability of lymphovascular invasion. The result of multivariate analysis showed that lymphangitic infiltration was the only independent factor correlating with the high fibrosis in FF in patients with IDC. Due to the missing data of clinicopathological features, only $49.7 \%$ cases with complete data were finally included in the ordinal logistic regression analysis, which might prevent us from discovering more factors correlating with the high fibrosis within FF in multivariate analysis. To sum up, the high fibrosis of FF can predict the strong invasiveness and high malignancy of breast IDC.

The relation between FF and the sub-factors of molecular subtypes is not very clear now. Hasebe et al (Hasebe et al. 2000; Hasebe et al. 1996) showed that FF was significantly correlated with HER-2 protein overexpression in IDC. Mujtaba et al (Mujtaba et al. 2013) revealed that FF was negatively associated with HER-2 expression $(P=0.021)$ and Ki-67 index $(P=0.001)$ and positively associated with HR expression $(P=0.007)$. However, Jeong et al (Jeong et al. 2018) did not find that FF was related to the expression of HR, HER-2, and Ki-67. The results of the studies above were controversial, which may be caused by the followings. Firstly, there were other histopathological types of breast cancer besides IDC, which were included in Mujtaba's and Jeong's studies. And the molecular subtypes of varying histopathological types were distinct in breast cancer. Moreover, the testing methods and the diagnostic criterion of HER-2 expression and Ki-67 index were different from the current ones. The association between FF and molecular subtypes is also unclear. Mujtab et al (Mujtaba et al. 2013) demonstrated that the FF was more common in Luminal A subtype than in non-Luminal A subtype $(\mathrm{n}=450, P<0.001)$. But another study (Jeong et al. 2018) did not find the association between FF and molecular subtypes ( $\mathrm{n}=$ 291, $P=0.830$ ). The different conclusions might be due to the inconsistencies of baseline 
281

282

283

284

285

286

287

288

289

290

291

292

293

294

295

296

297

298

299

300

301

302

303

304

305

306

307

308

309

310

311

312

313

314

315

characteristics of the cases included in the two studies. So more studies are needed in exploring the association between FF and molecular subtypes of breast cancer.

Few studies were conducted on the relation between the degree of fibrosis in FF and the subfactors of molecular subtypes of breast cancer. Only Hasebe et al (Hasebe et al. 1996) revealed that the HER-2 protein significantly overexpressed in the breast cancer with moderate and high fibrosis in FF (90.9\% vs. $41.7 \%, \mathrm{n}=153, P<0.02)$, compared with the breast cancer with mild fibrosis in FF. The similar conclusion was drawn in our study $(97.2 \%$ vs. $90.5 \%, \mathrm{n}=131, P=$ 0.032). Thus it can be seen that moderate and high fibrosis of FF is correlated with the invasiveness of breast cancer. Our study did not reveal any associations between the degree of fibrosis and other sub-factors of molecular subtypes including ER, PR and Ki-67 index. In addition, our result revealed that no association was found between the degree of fibrosis in FF and molecular subtypes. The relation between the degree of fibrosis in FF and molecular subtypes was not investigated in previous studies, so more studies are needed to explore the question.

The associations between FF and the survival of breast cancer have been reported in many studies. Hasebe et al (Hasebe et al. 1998) revealed that the presence of FF predicted higher risk of recurrence and death in breast cancer with less than four lymph nodes metastases or stage I $\sim$ I B B, compared with the absence of FF. Another study (Colpaert et al. 2001) including 104 cases of breast cancer with stage $\mathrm{T}_{1 \sim 2} \mathrm{~N}_{0} \mathrm{M}_{0}$ showed that the median disease-free survival (DFS) of cases with the presence of FF was significantly shorter than that with the absence of FF (25.0 months versus 91.5 months, $P<0.05$ ). Shimada et al (Shimada et al. 2017) demonstrated that the higher risk of recurrence (HR, 7.8; 95\%CI: 2.6-22.8; $P<0.001)$ and the shorter median progression-free survival (PFS) were found in breast cancer with the presence of FF. The studies above had indicated that the presence of FF was associated with the poor outcome of breast cancer. Therefore, FF was considered as a significant prognostic feature for breast cancer (Hasebe et al. 1998; Mujtaba et al. 2013). On the basis of previous studies, we further explored the correlation between the degree of fibrosis in FF and the long-term survival of breast IDC. All the cases included in our study had not been followed up to the median survival time. Thus, the short follow-up period led to the current inability of survival analysis, which was the greatest deficiency of our study. NPI was adopted as an alternative survival indicator to investigate the relation between the degree of fibrosis in FF and the long-term survival of breast cancer. NPI, reported first by Lee et al, is used widely to predict the 10-year overall survival (OS) rate of early breast cancer (Lee \& Ellis 2008). NPI is a scoring system of clinicopathology containing tumor size, histological grade, and lymph node stage. The NPI scores of 2.02 2.40, 2.41 3.40, $3.41 \sim 4.40,4.41 \sim 5.40,5.41 \sim 6.40$, and $6.41 \sim 6.80$ predict the 10-year overall survival rates of 
316

317

318

319

320

321

322

323

324

325

326

327

328

329

330

331

332

333

334

335

336

337

338

339

340

341

342

343

344

345

346

347

348

349

350

$96 \%, 93 \%, 81 \%, 74 \%, 55 \%$, and $38 \%$, respectively. According to the cutoffs of NPI score 3.40 and 5.40, the early breast cancer patients are stratified into good, moderate, and poor groups. The mean NPI in this study was $4.60 \pm 1.40$ and most cases were evaluated as moderate prognosis.

Our study revealed that the NPI of high fibrosis in FF showed an upward tendency, compared with that of mild and moderate fibrosis in $\mathrm{FF}(P=0.063)$. Further stratified analysis found that the NPI of high fibrosis in FF was significantly higher than that of mild and moderate fibrosis in FF in the no vascular infiltration subgroup, the no nerve infiltration subgroup, and the Luminal A subgroup. In general, the clinicopathological features of no vascular infiltration, no nerve infiltration, and Luminal A subtype indicate favorable outcomes of breast IDC. The presence of FF with high fibrosis could indicate the relatively worse outcomes of cases with the favourable clinicopathological features of breast IDC mentioned above, which was found in our study. Thus it can be seen that the degree of fibrosis in FF could be used as a practical and meaningful pathological feature for predicting the survival outcome of early breast IDC.

\section{Conclusions}

In summary, our study demonstrated that the high fibrosis in FF was closely associated with the strong invasiveness and the high malignancy of breast IDC. There are some disadvantages in our study, including the small sample size, partial clinicopathological data missing, and short follow-up period. In the future study, expanding the sample size, collecting sufficient clinicopathological data, and extending follow-up time should be considered.

\section{Acknowledgements}

We thank Doctor Rongxin Yan for assistance of collecting pathological data, and thank Doctor Xiangtao Lin for taking the pathological pictures.

\section{References}

Baak JP, Colpaert CG, van Diest PJ, Janssen E, van Diermen B, Albernaz E, Vermeulen PB, and Van Marck EA. 2005. Multivariate prognostic evaluation of the mitotic activity index and fibrotic focus in node-negative invasive breast cancers. Eur J Cancer 41:2093-2101 DOI: 10.1016/j.ejca.2005.03.038.

Balachander GM, Talukdar PM, Debnath M, Rangarajan A, and Chatterjee K. 2018. Inflammatory Role of Cancer-Associated Fibroblasts in Invasive Breast Tumors Revealed Using a Fibrous Polymer Scaffold. ACS Appl Mater Interfaces 10:3381433826 DOI: 10.1021 acsami.8b07609.

Colpaert C, Vermeulen P, van Beest P, Goovaerts G, Weyler J, Van Dam P, Dirix L, and Van 
351

352

353

354

355

356

357

358

359

360

361

362

363

364

365

366

367

368

369

370

371

372

373

374

375

376

377

378

379

380

381

382

383

384

385

386

387

388

389

390

391

Marck E. 2001. Intratumoral hypoxia resulting in the presence of a fibrotic focus is an independent predictor of early distant relapse in lymph node-negative breast cancer patients. Histopathology 39:416-425.

Daniel SK, Sullivan KM, Labadie KP, and Pillarisetty VG. 2019. Hypoxia as a barrier to immunotherapy in pancreatic adenocarcinoma. Clin Transl Med 8:10 DOI: 10.1186/s40169-019-0226-9.

DeSantis CE, Ma J, Goding Sauer A, Newman LA, and Jemal A. 2017. Breast cancer statistics, 2017, racial disparity in mortality by state. CA Cancer J Clin 67:439-448 DOI: $10.3322 /$ caac. 21412 .

Eiro N, Gonzalez L, Martinez-Ordonez A, Fernandez-Garcia B, Gonzalez LO, Cid S, Dominguez F, Perez-Fernandez R, and Vizoso FJ. 2018. Cancer-associated fibroblasts affect breast cancer cell gene expression, invasion and angiogenesis. Cell Oncol (Dordr) 41:369-378 DOI: 10.1007/s13402-018-0371-y.

Elston EW, and Ellis IO. 1993. Method for grading breast cancer. J Clin Pathol 46:189-190.

Goldhirsch A, Wood WC, Coates AS, Gelber RD, Thurlimann B, Senn HJ, and Panel m. 2011. Strategies for subtypes--dealing with the diversity of breast cancer: highlights of the St. Gallen International Expert Consensus on the Primary Therapy of Early Breast Cancer 2011. Ann Oncol 22:1736-1747 DOI: 10.1093/annonc/mdr304.

Grasso C, Jansen G, and Giovannetti E. 2017. Drug resistance in pancreatic cancer: Impact of altered energy metabolism. Crit Rev Oncol Hematol 114:139-152 DOI: 10.1016/j.critrevonc.2017.03.026.

Hasebe T, Mukai K, Tsuda H, and Ochiai A. 2000. New prognostic histological parameter of invasive ductal carcinoma of the breast: clinicopathological significance of fibrotic focus. Pathol Int 50:263-272.

Hasebe T, Sasaki S, Imoto S, Mukai K, Yokose T, and Ochiai A. 2002. Prognostic significance of fibrotic focus in invasive ductal carcinoma of the breast: a prospective observational study. Mod Pathol 15:502-516 DOI: 10.1038/modpathol.3880555.

Hasebe T, Tsuda H, Hirohashi S, Shimosato Y, Iwai M, Imoto S, and Mukai K. 1996. Fibrotic focus in invasive ductal carcinoma: an indicator of high tumor aggressiveness. Jpn J Cancer Res 87:385-394.

Hasebe T, Tsuda H, Hirohashi S, Shimosato Y, Tsubono Y, Yamamoto H, and Mukai K. 1998. Fibrotic focus in infiltrating ductal carcinoma of the breast: a significant histopathological prognostic parameter for predicting the long-term survival of the patients. Breast Cancer Res Treat 49:195-208.

Hasebe T, Tsuda H, Tsubono Y, Imoto S, and Mukai K. 1997. Fibrotic focus in invasive ductal carcinoma of the breast: a histopathological prognostic parameter for tumor recurrence and tumor death within three years after the initial operation. Jpn J Cancer Res 88:590599.

Hoffmann C, Mao X, Brown-Clay J, Moreau F, Al Absi A, Wurzer H, Sousa B, Schmitt F, Berchem G, Janji B, and Thomas C. 2018. Hypoxia promotes breast cancer cell invasion through HIF-1alpha-mediated up-regulation of the invadopodial actin bundling protein 
CSRP2. Sci Rep 8:10191 DOI: 10.1038/s41598-018-28637-x.

Ikuta D, Miyake T, Shimizu T, Sonoda H, Mukaisho KI, Tokuda A, Ueki T, Sugihara H, and Tani M. 2018. Fibrosis in metastatic lymph nodes is clinically correlated to poor prognosis in colorectal cancer. Oncotarget 9:29574-29586 DOI: 10.18632/oncotarget.25636.

Jeong YJ, Park SH, Mun SH, Kwak SG, Lee SJ, and Oh HK. 2018. Association between lysyl oxidase and fibrotic focus in relation with inflammation in breast cancer. Oncol Lett 15:2431-2440 DOI: 10.3892/ol.2017.7617.

Kornegoor R, Verschuur-Maes AH, Buerger H, Hogenes MC, de Bruin PC, Oudejans JJ, Hinrichs B, and van Diest PJ. 2012. Fibrotic focus and hypoxia in male breast cancer. Mod Pathol 25:1397-1404 DOI: 10.1038/modpathol.2012.101.

Lee AH, and Ellis IO. 2008. The Nottingham prognostic index for invasive carcinoma of the breast. Pathol Oncol Res 14:113-115 DOI: 10.1007/s12253-008-9067-3.

Maiorano E, Regan MM, Viale G, Mastropasqua MG, Colleoni M, Castiglione-Gertsch M, Price KN, Gelber RD, Goldhirsch A, and Coates AS. 2010. Prognostic and predictive impact of central necrosis and fibrosis in early breast cancer: results from two International Breast Cancer Study Group randomized trials of chemoendocrine adjuvant therapy. Breast Cancer Res Treat 121:211-218 DOI: 10.1007/s10549-009-0360-y.

Mujtaba SS, Ni YB, Tsang JY, Chan SK, Yamaguchi R, Tanaka M, Tan PH, and Tse GM. 2013. Fibrotic focus in breast carcinomas: relationship with prognostic parameters and biomarkers. Ann Surg Oncol 20:2842-2849 DOI: 10.1245/s10434-013-2955-0.

Plichta JK, Ren Y, Thomas SM, Greenup RA, Fayanju OM, Rosenberger LH, Hyslop T, and Hwang ES. 2018. Implications for Breast Cancer Restaging Based on the 8th Edition AJCC Staging Manual. Ann Surg. DOI: 10.1097/SLA.0000000000003071.

Prat A, Cheang MC, Martin M, Parker JS, Carrasco E, Caballero R, Tyldesley S, Gelmon K, Bernard PS, Nielsen TO, and Perou CM. 2013. Prognostic significance of progesterone receptor-positive tumor cells within immunohistochemically defined luminal A breast cancer. J Clin Oncol 31:203-209 DOI: 10.1200/JCO.2012.43.4134.

Reisfeld RA. 2013. The tumor microenvironment: a target for combination therapy of breast cancer. Crit Rev Oncog 18:115-133.

Salmon H, Franciszkiewicz K, Damotte D, Dieu-Nosjean MC, Validire P, Trautmann A, MamiChouaib F, and Donnadieu E. 2012. Matrix architecture defines the preferential localization and migration of $\mathrm{T}$ cells into the stroma of human lung tumors. J Clin Invest 122:899-910 DOI: 10.1172/JCI45817.

Shimada H, Hasebe T, Sugiyama M, Shibasaki S, Sugitani I, Ueda S, Gotoh Y, Yasuda M, Arai E, Osaki A, and Saeki T. 2017. Fibrotic focus: An important parameter for accurate prediction of a high level of tumor-associated macrophage infiltration in invasive ductal carcinoma of the breast. Pathol Int 67:331-341 DOI: 10.1111/pin.12550.

Thomas D, and Radhakrishnan P. 2019. Tumor-stromal crosstalk in pancreatic cancer and tissue fibrosis. Mol Cancer 18:14 DOI: 10.1186/s12943-018-0927-5.

Van den Eynden GG, Colpaert CG, Couvelard A, Pezzella F, Dirix LY, Vermeulen PB, Van 
433

434

435

436

437

438

439

440

441

442

443

444

445

446

447

448

449

450

451

452

Marck EA, and Hasebe T. 2007. A fibrotic focus is a prognostic factor and a surrogate marker for hypoxia and (lymph)angiogenesis in breast cancer: review of the literature and proposal on the criteria of evaluation. Histopathology 51:440-451 DOI: $10.1111 / \mathrm{j} .1365-$ 2559.2007.02761.x.

Van den Eynden GG, Smid M, Van Laere SJ, Colpaert CG, Van der Auwera I, Bich TX, van Dam P, den Bakker MA, Dirix LY, Van Marck EA, Vermeulen PB, and Foekens JA. 2008. Gene expression profiles associated with the presence of a fibrotic focus and the growth pattern in lymph node-negative breast cancer. Clin Cancer Res 14:2944-2952 DOI: 10.1158/1078-0432.CCR-07-4397.

Wolff AC, Hammond ME, Hicks DG, Dowsett M, McShane LM, Allison KH, Allred DC, Bartlett JM, Bilous M, Fitzgibbons P, Hanna W, Jenkins RB, Mangu PB, Paik S, Perez EA, Press MF, Spears PA, Vance GH, Viale G, Hayes DF, American Society of Clinical O, and College of American P. 2013. Recommendations for human epidermal growth factor receptor 2 testing in breast cancer: American Society of Clinical Oncology/College of American Pathologists clinical practice guideline update. J Clin Oncol 31:3997-4013 DOI: 10.1200/JCO.2013.50.9984.

Yang X, Lin Y, Shi Y, Li B, Liu W, Yin W, Dang Y, Chu Y, Fan J, and He R. 2016. FAP Promotes Immunosuppression by Cancer-Associated Fibroblasts in the Tumor Microenvironment via STAT3-CCL2 Signaling. Cancer Res 76:4124-4135 DOI: 10.1158/0008-5472.CAN-15-2973. 


\section{Figure 1}

Representative histology of fibrotic focus (FF) in breast invasive ductal carcinoma (IDC).

(A) A representative histology of FF with the appearance of scar-like lesion indicated by arrows [H\&E; magnification, $\times 20]$. (B) A representative histology of FF with the appearance of irregular moth-eaten radiating fibrosclerotic core indicated by arrows [H\&E; magnification, $\times 20$ ]. (C) FF with mild fibrosis showing high number of fibroblasts and small amount of collagen fibers in stroma [H\&E; magnification, $\times 100]$. (D) FF with moderate fibrosis intermediating between mild fibrosis and high fibrosis and numerous tumor nests in $\mathrm{FF}$ [H\&E; magnification, $\times 100]$. (E) FF with high fibrosis showing mostly hyalinized collagen fibers [H\&E; magnification, $\times 100$ ]. (F) High fibrosis and peripheral vascular invasion indicated by the arrow [H\&E; magnification, $\times 100]$. Photograph credit: Doctor Xiangtao Lin. 

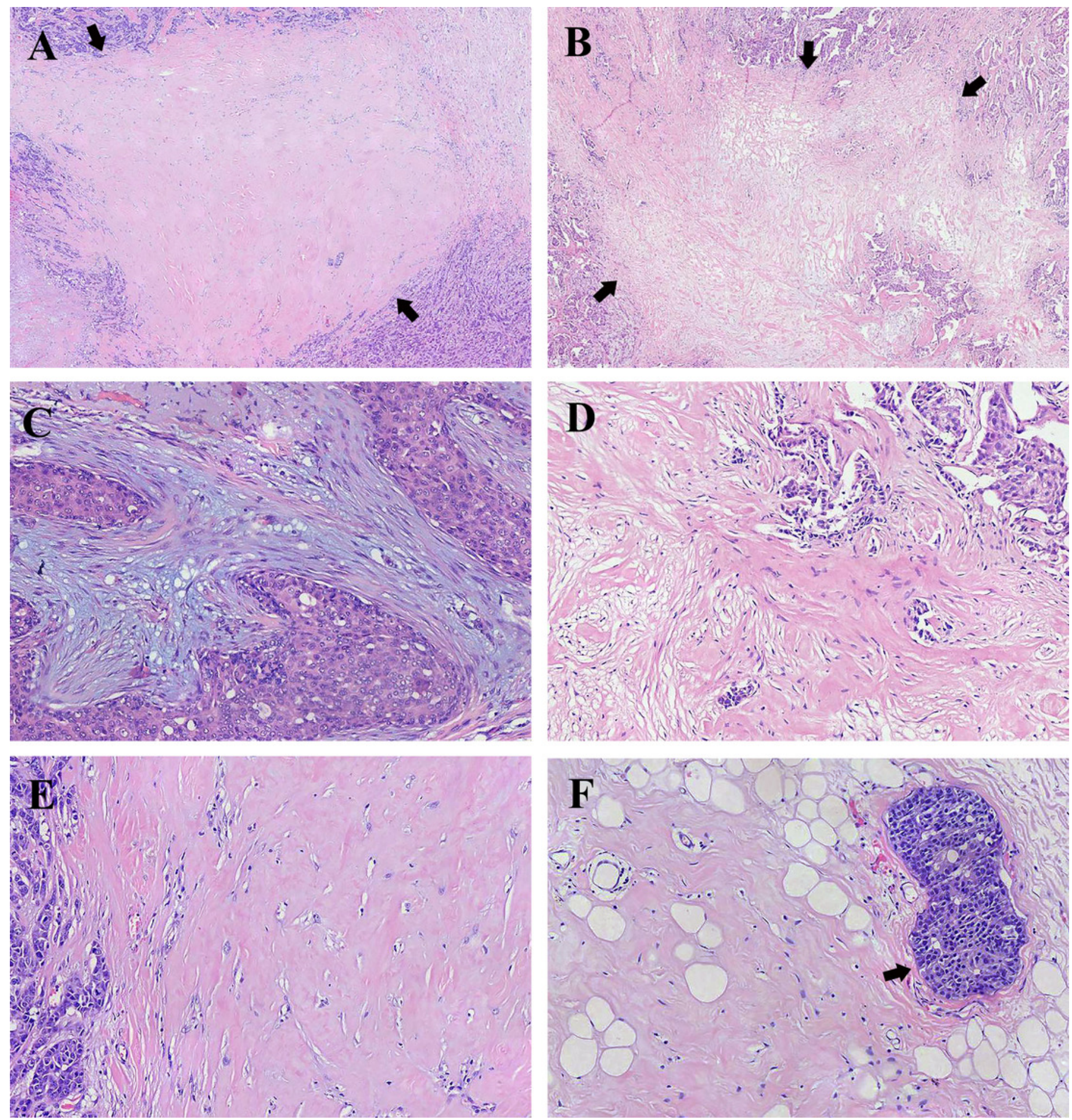


\section{Figure 2}

The distribution of different degrees of fibrosis in FF in different clinicopathologic features.

(A) The comparison of the proportions of different degrees of fibrosis in FF between $T \leq 2 \mathrm{~cm}$

$\left(T_{1}\right)$ and $T>2 \mathrm{~cm}\left(T_{2 \sim 3}\right)$. (B) The comparison of the proportions of different degrees of fibrosis

in FF between $\mathrm{VI}$ - and $\mathrm{VI}+$. (C) The comparison of the proportions of different degrees of fibrosis in FF between LVI- and LVI+. (D) The comparison of the proportions of different degrees of fibrosis in FF between HER-2- and HER-2+. Notes: $\mathrm{T}=$ tumor size; $\mathrm{VI}-\mathrm{=}$ no vascular invasion; $\mathrm{VI}+=$ vascular invasion; $\mathrm{LVI}-=$ no lymphatic vessel invasion; $\mathrm{LVI}+=$ lymphatic vessel invasion. 


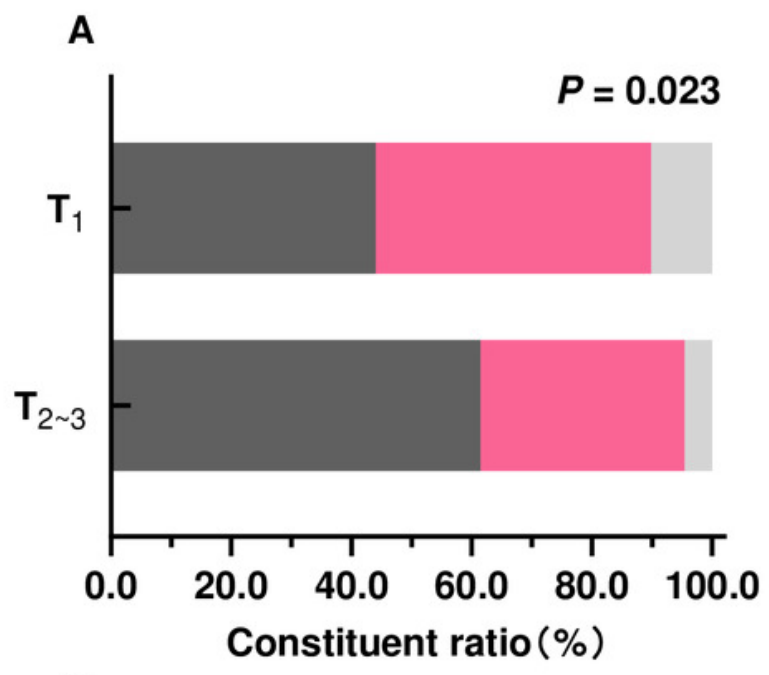

C
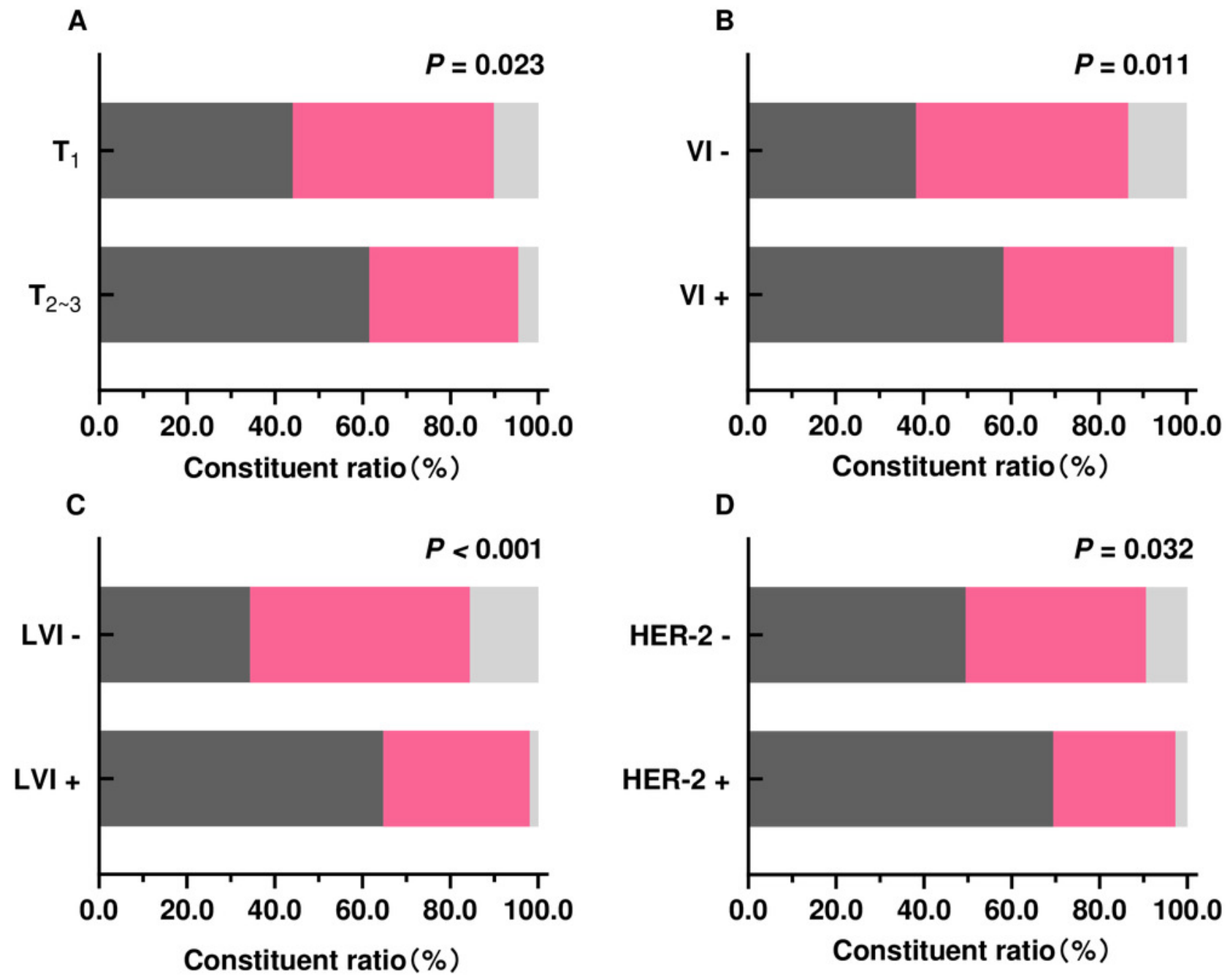

Mild

Moderate

High 
Figure 3

The comparison of NPI variables between mild and moderate fibrosis in FF and high fibrosis in FF.

Notes: $\mathrm{VI}-=$ no vascular invasion; $\mathrm{Nl}-=$ no nervous infiltration.

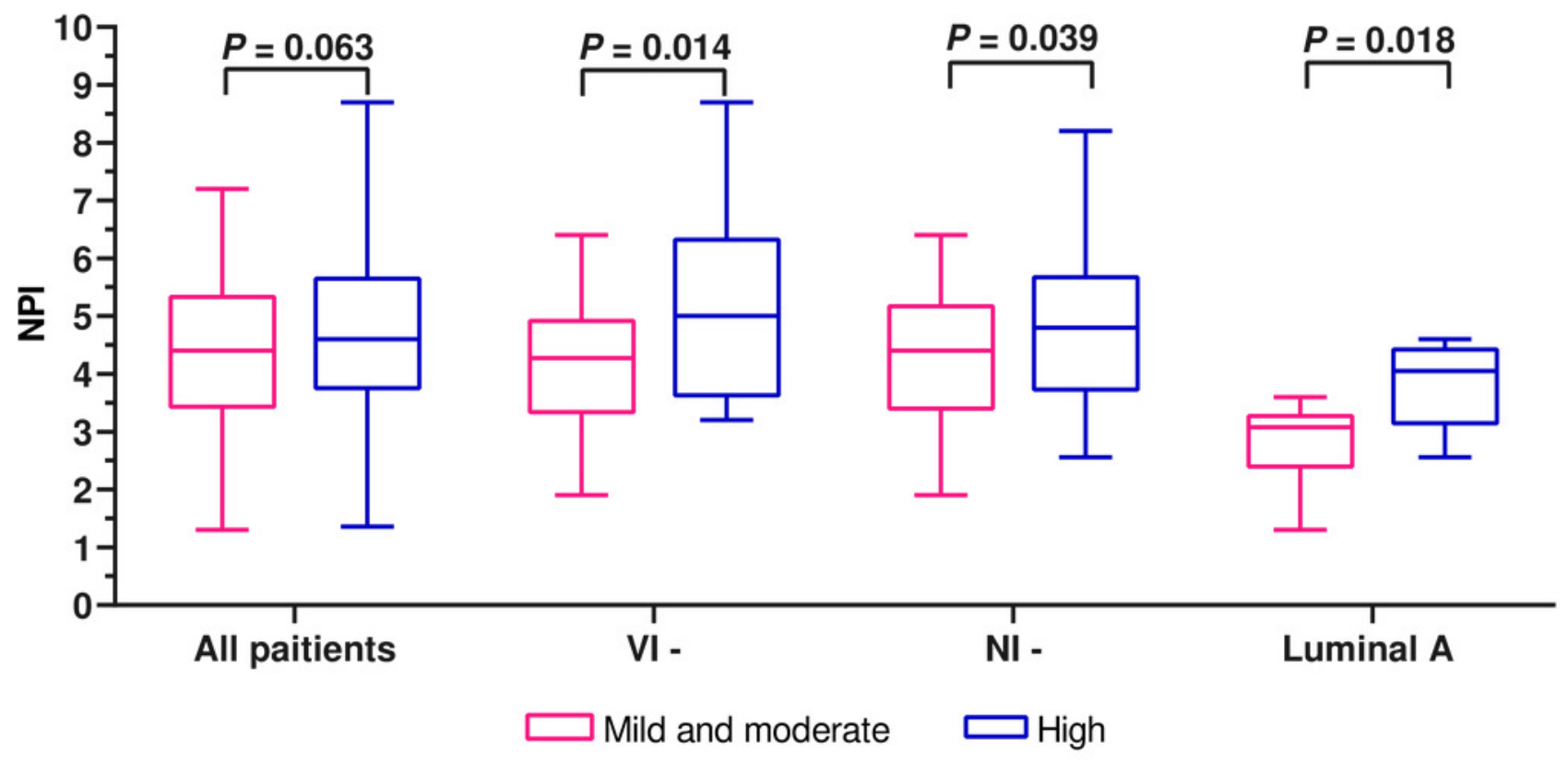


Table $\mathbf{1}$ (on next page)

The characteristics of patients 
1 Table 1:

2 The characteristics of patients

\begin{tabular}{|c|c|c|c|}
\hline Clinicopathologic & & Clinicopathologic & \\
\hline & n $(\%)$ & & n $(\%)$ \\
\hline variables & \multicolumn{3}{|c|}{ variables } \\
\hline Age(years) & \multicolumn{3}{|c|}{ Nervous infiltration } \\
\hline$\leq 50$ & $79(46.7)$ & Yes & 32 (18.9) \\
\hline$>50$ & $90(53.3)$ & No & $81(47.9)$ \\
\hline Tumor size & & Unknown & $56(33.2)$ \\
\hline$\leq 2 \mathrm{~cm}$ & $59(34.9)$ & $\mathbf{E R}$ & \\
\hline$>2 \mathrm{~cm}$ & $109(64.5)$ & Positive & $108(63.9)$ \\
\hline Unknown & $1(0.6)$ & Negative & $60(35.5)$ \\
\hline Lymph node & & Unknown & $1(0.6)$ \\
\hline metastasis & & PR & \\
\hline Yes & $90(53.3)$ & Positive & $100(59.1)$ \\
\hline No & $76(45.0)$ & Negative & $68(40.2)$ \\
\hline Unknown & $3(1.7)$ & Unknown & $1(0.7)$ \\
\hline stage & & Ki-67 & \\
\hline I & $34(20.1)$ & $\leq 14 \%$ & $35(20.7)$ \\
\hline II & $87(51.5)$ & $>14 \%$ & $129(76.3)$ \\
\hline III & $44(26.0)$ & Unknown & $5(3.0)$ \\
\hline
\end{tabular}


4

\begin{tabular}{|c|c|c|c|}
\hline Histological grade & & Positive & $36(21.3)$ \\
\hline $\mathrm{I} \sim \mathrm{II}$ & $88(52.1)$ & Negative & $95(56.2)$ \\
\hline III & $62(36.7)$ & Unknown & $38(22.5)$ \\
\hline Unknown & $19(11.2)$ & Molecular subtype & \\
\hline Vascular invasion & & Luminal A & $16(9.5)$ \\
\hline Yes & $67(39.6)$ & Luminal B & $93(55.0)$ \\
\hline No & $60(35.5)$ & HER-2 & \\
\hline Unknown & $42(24.9)$ & overexpression & $17(10.1)$ \\
\hline Lymphatic vessel & & TNBC & $24(14.2)$ \\
\hline invasion & & Unknown & $19(11.2)$ \\
\hline Yes & $51(30.1)$ & & \\
\hline No & $64(37.9)$ & & \\
\hline Unknown & $54(32.0)$ & & \\
\hline
\end{tabular}




\section{Table 2 (on next page)}

The correlation between clinicopathological characteristics and degree of fibrosis in FF

"Mann-whitney U test " Jonckheere-Terpstra test 
1 Table 2:

2 The correlation between clinicopathological characteristics and degree of fibrosis in FF

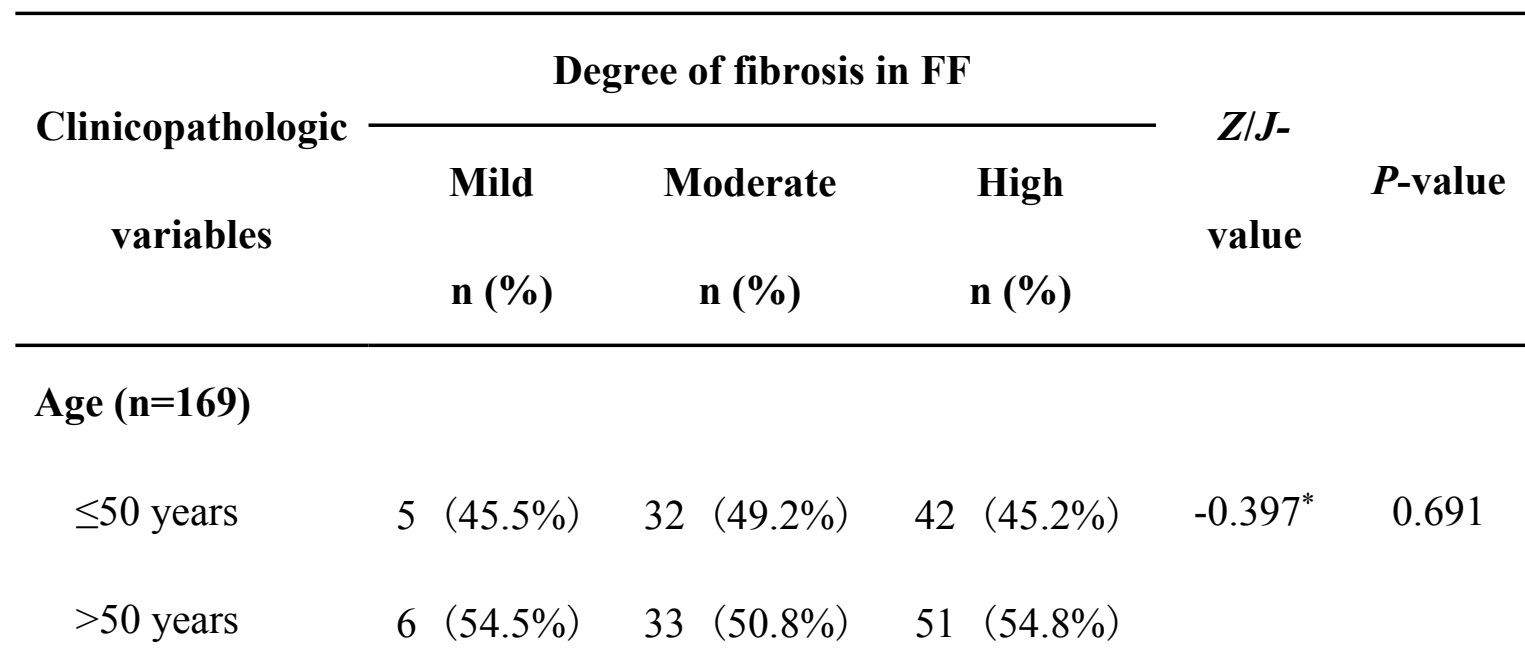

Tumor size
$(\mathbf{n}=168)$

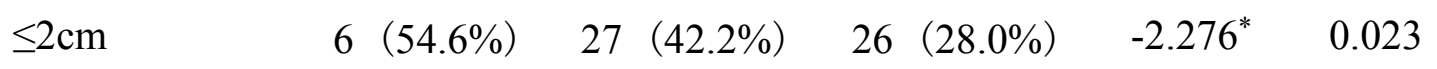
$>2 \mathrm{~cm} \quad 5(45.4 \%) \quad 37(57.8 \%) \quad 67(72.0 \%)$

Lymph node

metastasis

$(n=166)$

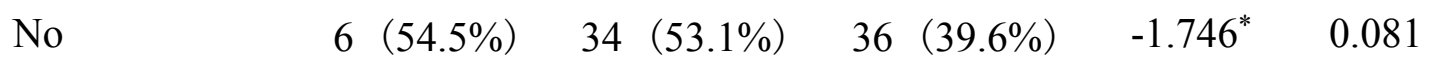

Yes $\quad 5(45.5 \%) \quad 30(46.9 \%) \quad 55(60.4 \%)$

stage $(\mathbf{n}=165)$

$\begin{array}{lllllll}\text { I } & 4(36.4 \%) & 16(25.4 \%) & 14(15.4 \%) & 1.921^{\#} & 0.055\end{array}$

II $\quad 4(36.4 \%) \quad 34(54.0 \%) \quad 49(53.8 \%)$ 
III

$3(27.2 \%) \quad 13(20.6 \%) \quad 28(30.8 \%)$

\section{Histological}

grade $(n=150)$
I $~ I I$
$4(40.0 \%)$
$40(67.8 \%)$
$44(54.3 \%)$
$-0.786^{*}$
0.432
III
$6(60.0 \%) \quad 19(32.2 \%) \quad 37(45.6 \%)$

Vascular

invasion $(n=127)$

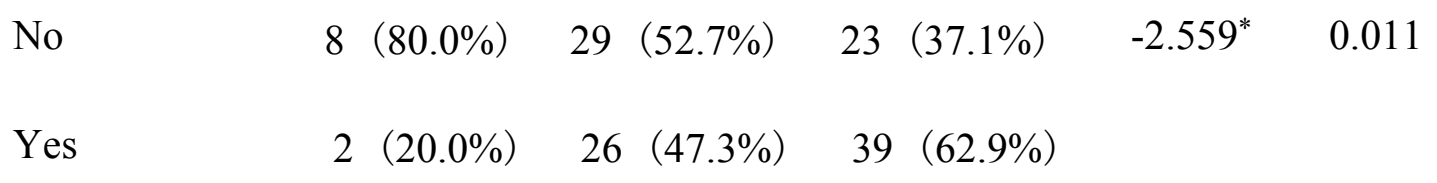

\section{Lymphatic vessel}

invasion $(\mathrm{n}=115)$

No

$10(90.9 \%)$

$32(65.3 \%)$

$22(40.0 \%)$

$-3.523^{*}$

$<0.001$

Yes

$1(9.1 \%)$

$17(34.7 \%)$

$33(60.0 \%)$

nervous

infiltration

$$
(n=113)
$$

No

$9(81.8 \%)$

$38(76.0 \%)$

$34(65.4 \%) \quad-1.419^{*}$

0.156

Yes

$2(18.2 \%)$

$12(24.0 \%)$

$18(34.6 \%)$

3 "Mann-whitney U test

4 \# Jonckheere-Terpstra test 


\section{Table 3(on next page)}

The association analysis of different variables between molecular subtypes and degree of fibrosis in $\mathrm{FF}$

"Mann-whitney U test ${ }^{*}$ Jonckheere-Terpstra test 


\section{Table 3:}

2 The association analysis of different variables between molecular subtypes and degree of 3 fibrosis in FF

\begin{tabular}{|c|c|c|c|c|c|}
\hline \multirow{3}{*}{ Variables } & \multicolumn{3}{|c|}{ Degree of fibrosis in FF } & \multirow{2}{*}{$Z / J-$} & \multirow[b]{2}{*}{$P$-value } \\
\hline & Mild & Moderate & High & & \\
\hline & n (\%) & n (\%) & n (\%) & & \\
\hline
\end{tabular}

$\operatorname{ER}(\mathbf{n}=168)$

Negative $\quad 6(54.6 \%) \quad 20(31.2 \%) \quad 34(36.6 \%) \quad-0.060^{*} \quad 0.952$

Positive $\quad 5(45.4 \%) \quad 44(68.8 \%) \quad 59(63.4 \%)$

$\operatorname{PR}(\mathbf{n}=168)$

$\begin{array}{llllll}\text { Negative } & 7(63.6 \%) & 22(34.4 \%) & 39(41.9 \%) & -0.040^{*} & 0.968 \\ \text { Positive } & 4(36.4 \%) & 42(65.6 \%) & 54(58.1 \%) & & \\ \text { Ki-67 (n=164) } & & & & & \\ \leq 14 \% & 3(27.3 \%) & 16(26.2 \%) & 16(17.4 \%) & -1.377^{*} & 0.168 \\ >14 \% & 8(72.7 \%) & 45(73.8 \%) & 76(82.6 \%) & & \end{array}$

HER-2 (n=131)

$\begin{array}{llllll}\text { Negative } & 9(90.0 \%) & 39(79.6 \%) & 47(65.3 \%) & -2.141^{*} & 0.032\end{array}$

Positive $\quad 1(10.0 \%) \quad 10(20.4 \%) \quad 25(34.7 \%)$

Molecular

subtypes 


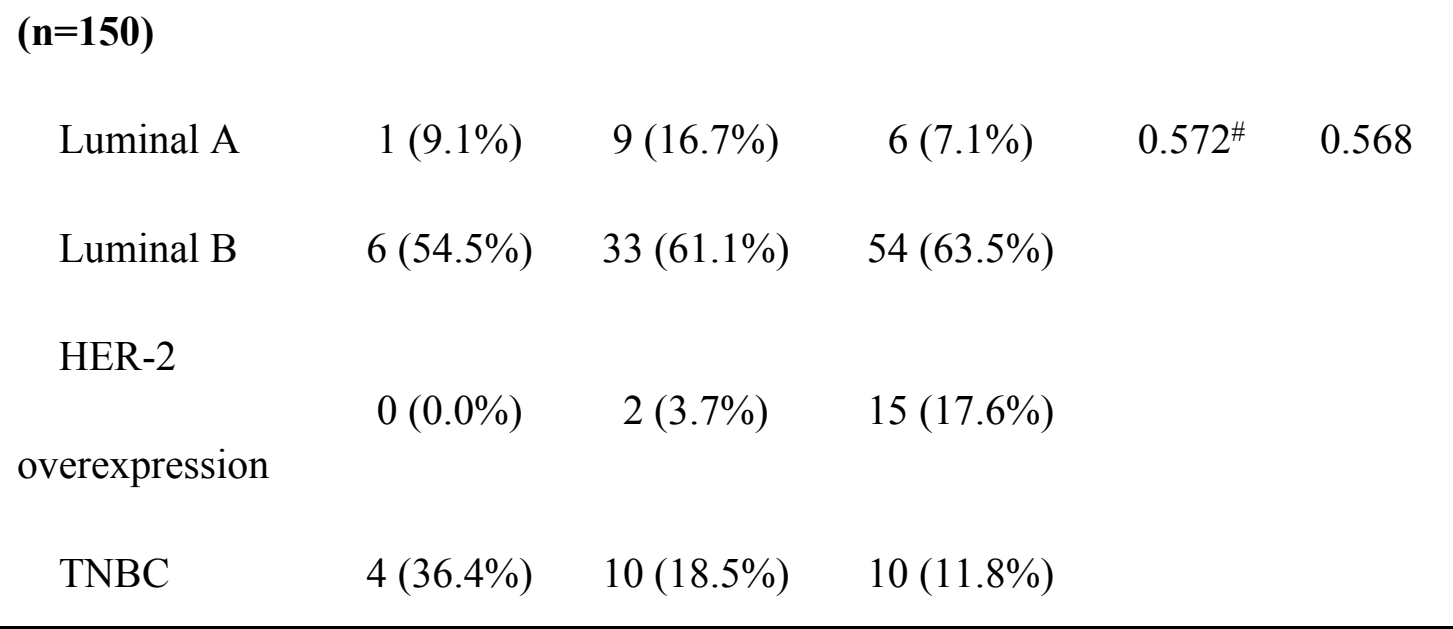

$4 \quad$ *Mann-whitney U test

$5 \quad$ \# Jonckheere-Terpstra test 


\section{Table 4 (on next page)}

The comparison of NPI variables between mild and moderate fibrosis in FF and high fibrosis in FF after stratification

"Mann-whitney $U$ test ${ }^{\#} 5.15$ was the median NPI. Since there were only 2 cases, the $P_{25}$ and $P_{75}$ could not be calculated. 
1 Table 4:

2 The comparison of NPI variables between mild and moderate fibrosis in FF and high

3 fibrosis in FF after stratification

\begin{tabular}{|c|c|c|c|c|}
\hline \multirow{3}{*}{ Stratification } & \multicolumn{2}{|c|}{ Degree of fibrosis in FF } & \multirow{3}{*}{$\begin{array}{c}Z \text { - } \\
\text { Value* }\end{array}$} & \multirow{3}{*}{$\begin{array}{c}P \text { - } \\
\text { value }\end{array}$} \\
\hline & \multirow{2}{*}{$\begin{array}{l}\text { Mild and moderate } \\
\qquad P_{25} 、 M 、 P_{75}(n)\end{array}$} & \multirow[b]{2}{*}{$P_{25} 、 \mathrm{M} 、 P_{75}(\mathrm{n})$} & & \\
\hline & & & & \\
\hline \multicolumn{5}{|l|}{ Age (years) } \\
\hline$\leq 50$ & $3.36,4.50,5.40(37)$ & $3.36,4.50,5.40(37)$ & -0.930 & 0.352 \\
\hline$>50$ & $3.40,4.40,5.20(36)$ & $4.30,4.70,5.60(51)$ & -1.690 & 0.091 \\
\hline
\end{tabular}

Tumor size

$\begin{array}{lllll}\leq 2 \mathrm{~cm} & 3.30,4.27,5.20(32) & 3.33,4.26,4.90(25) & -0.299 & 0.765 \\ & 3.60,4.60,5.60(41) & 4.50,5.00,5.80(63) & -1.448 & 0.148\end{array}$

Lymph node

metastasis

$\begin{array}{lllll}\text { No } & 3.29,3.50,4.40(38) & 3.30,3.70,4.50(35) & -0.696 & 0.486 \\ \text { Yes } & 4.60,5.36,5.90(35) & 4.60,5.60,6.35(53) & -1.063 & 0.288\end{array}$

stage

$\begin{array}{lllll}\text { I } & 3.21,3.36,4.29(20) & 3.30,3.40,4.27(14) & -0.406 & 0.691 \\ \text { II } & 3.58,4.50,5.15(37) & 3.70,4.54,5.00(47) & -0.465 & 0.642 \\ \text { III } & 5.32,5.80,6.30(16) & 5.60,6.30,6.80(27) & -1.436 & 0.151\end{array}$




\section{Histological grade}

$\begin{array}{lllll}\text { I } \sim \text { II } & 3.32,3.70,5.00(43) & 3.50,4.50,5.40(43) & -1.517 & 0.129 \\ \text { III } & 4.50,5.20,5.60(25) & 4.57,5.60,6.45(37) & -1.443 & 0.149\end{array}$

Vascular invasion

No

Yes

\section{Lymphatic vessel}

invasion

$$
\begin{array}{llll}
3.31,4.27,4.95(36) & 3.60,5.00,6.35(21) & -2.458 & 0.014 \\
& & & \\
3.58,5.10,5.70(26) & 4.31,4.93,5.85(38) & -0.616 & 0.538
\end{array}
$$

\section{invasion}

$\begin{array}{lllll}\text { No } & 3.36,4.40,5.18(40) & 3.50,5.00,6.00(21) & -1.709 & 0.087 \\ \text { Yes } & 3.58,4.56,5.70(17) & 4.46,5.18,5.80(32) & -1.441 & 0.150\end{array}$

\section{Nervous infiltration}

$\begin{array}{lllll}\text { No } & 3.37,4.40,5.20(45) & 3.70,4.80,5.70(32) & -2.063 & 0.039 \\ \text { Yes } & 3.35,4.80,5.65(13) & 4.43,5.20,6.53(16) & -0.430 & 0.446\end{array}$

ER

$\begin{array}{lllll}\text { Negative } & 3.68,5.00,5.43(26) & 4.53,5.40,6.30(32) & -1.799 & 0.072 \\ \text { Positive } & 3.30,4.40,5.00(47) & 3.50,4.47,5.60(56) & -1.060 & 0.289\end{array}$

PR

$\begin{array}{lllll}\text { Negative } & 4.28,4.68,5.39(28) & 4.57,5.10,6.20(37) & -1.955 & 0.051 \\ \text { Positive } & 3.30,3.90,5.25(45) & 3.40,4.40,5.60(51) & -0.962 & 0.336\end{array}$

\section{Ki-67}




$\begin{array}{lllll}\leq 14 \% & 2.77,3.40,4.42(17) & 3.33,3.70,4.58(16) & -1.353 & 0.179 \\ >14 \% & 3.65,4.60,5.40(53) & 4.26,4.96,5.70(71) & -1.519 & 0.129\end{array}$

HER-2

$\begin{array}{lllll}\text { Negative } \quad 3.39,4.40,5.32(46) & 3.70,4.50,5.70(43) & -1.450 & 0.147\end{array}$

Positive $\quad 4.56,5.20,5.70(11) \quad 4.32,5.05,5.80(24) \quad-0.124 \quad 0.903$

\section{Molecular subtypes}

Luminal A

Luminal B

HER-2

$$
2.37,3.08,3.30(9) \quad 3.12,4.05,4.45(6) \quad-2.359 \quad 0.018
$$$$
3.48,4.58,5.43(38) \quad 3.70,4.50,5.60(51) \quad-0.183 \quad 0.855
$$

$$
5.15^{\#}(2)
$$

$4.93,5.70,6.58(14)$

$-0.954$

0.417

overexpression 